\title{
PRODUCING THE TANGENCY PORTFOLIO AS A CORNER PORTFOLIO
}

\author{
Reza Keykhaei ${ }^{1}$ And Mohamad-Taghi JahandideH ${ }^{2}$
}

\begin{abstract}
One-fund theorem states that an efficient portfolio in a Mean-Variance (M-V) portfolio selection problem for a set of some risky assets and a riskless asset can be represented by a combination of a unique risky fund (tangency portfolio) and the riskless asset. In this paper, we introduce a method for which the tangency portfolio can be produced as a corner portfolio. So, the tangency portfolio can be computed easily and fast by any algorithm designed for tracing out the $\mathrm{M}-\mathrm{V}$ efficient frontier via computing the corner portfolios. Moreover, we show that how this method can be used for tracing out the M-V efficient frontier when problem contains a riskless asset in which the borrowing is not allowed.
\end{abstract}

Keywords. M-V Optimization, Parametric Quadratic Programming, Critical Line Algorithm, Capital Allocation Line, Tangency Portfolio.

Mathematics Subject Classification. 91G10, 90C20, 90C29.

\section{INTRODUCTION}

The aim of classic Mean-Variance (M-V) portfolio optimization, originated from the seminal work of Markowitz [7], is to maximize the expected return of a portfolio and minimize its variance as the measure of risk. Markowitz proposed his work for a set of risky assets. M-V portfolio selection problems seek to compute efficient portfolios. A portfolio is efficient if with respect to its location in the M-V space, there is no obtainable portfolio with a lower variance without a lower expected return or a greater expected return without a greater variance. The locus of all

Received July 4, 2012. Accepted July 11, 2013.

1 Department of Mathematics, Khansar Faculty of Computer and Mathematics, University of Isfahan, Isfahan 81746-73441, Iran. r.keykhaei@math.iut.ac.ir

2 College of Mathematical Sciences, Isfahan University of Technology, 84156-83111, Isfahan, Iran. jahandid@cc.iut.ac.ir 
efficient portfolios in the M-V plane is called the efficient frontier. The idea of riskless asset was first suggested by Tobin [16]. He included cash in his version of portfolio selection problem and stated that any efficient portfolio is a combination of a single risky fund and the riskless asset. This is the Tobin's one-fund theorem. In fact all combinations of a risky portfolio and a riskless asset can be represented by a line, Capital Allocation Line (CAL), originating at the riskless asset and passing through the risky portfolio, in the Mean-Standard Deviation (M-SD) plane. There exists a CAL termed by optimal CAL, which dominates the other CALs. When borrowing of riskless asset is allowed, the efficient frontier is the optimal CAL. The optimal CAL has the highest possible slop and is tangent to the efficient frontier of risky assets. We denote the risky portfolio corresponding to the tangent point by the tangency portfolio. Indeed tangency portfolio is the efficient portfolio which maximizes the famous Sharpe ratio [14]:

$$
\frac{\rho-r_{f}}{\sigma}
$$

where, $\rho$ and $\sigma$ denotes the mean and the standard deviation of any efficient portfolio, respectively, and $r_{f}$ denotes the return of the riskless asset. So, in order to find the tangency portfolio it is enough to find efficient portfolios and recognize the tangency portfolio which maximizes the Sharpe ratio (for example see chapter seven of [3]). Markowitz [8] proposed his Critical Line Algorithm (CLA), as a Parametric Quadratic Programming (PQP), for general portfolio selection models and developed it in his books [9-11], for computing the efficient portfolios. Jacobs et al. [6] extended CLA to account for factor and scenario models with realistic short positions. In addition to Markowits's algorithm, there are other $\mathrm{PQP}$ algorithms which proposed in the literature to trace out the M-V efficient frontier, for example we can refer to Best [1], Stein et al. [15], Niedermayer and Niedermayer [13] and Hirschberger et al. [5]. As we know, in the M-V plane, the efficient frontier is consisting of connected parabolic segments. The portfolios corresponding to the end points of each segment are called corner portfolios. The major aim of the above works is to introduce methods which calculate the corner portfolios and the efficient frontier while significantly reducing computational time.

Todd programmed CLA algorithm in Visual Basic for Applications (VBA) and the software is called Optimizer (see Markowitz and Todd [11]). Niedermayer and Niedermayer [13] provide a Matlab quadratic optimization tool based on Markowitz's CLA. Their method computes the efficient frontier of the standard problem with 2000 assets in less than a second.

Tütüncü [17] presented a modification of the CLA which computes the tangency portfolio as a by-product during the algorithm. In this paper, we present an algorithm, based on Tütüncü's results, for which the tangency portfolio can be produced as a corner portfolio. So, the tangency portfolio, as a corner portfolio, can be calculated in a short time using CLA (or other suitable methods). Moreover, we show that how this method can be used to trace out the efficient frontier 
when portfolio contains a riskless asset and borrowing is not allowed for riskless asset.

The paper proceeds is as follows. The M-V portfolio selection problem formulation and the results of Tütüncü about the tangency portfolio are described in Section 2. In Section 3 we describe the modified portfolio selection problem and give the main results. in Section 4 we present our algorithm for finding the tangency portfolio.

\section{The tangency PortFolio}

Consider a portfolio consisting of $n \geq 2$ risky assets with the random returns $r_{1}, \ldots, r_{n}$. Let $\overline{\mathbf{R}}=\left(\bar{r}_{1}, \ldots, \bar{r}_{n}\right)^{\prime}$ and $\boldsymbol{\Sigma}$ be the mean vector and covariance matrix of the asset returns, respectively. We denote each portfolio by the vector of asset weights $\mathbf{x}:=\left(x_{1}, \ldots, x_{n}\right)^{\prime} \in \mathbb{R}^{n}$, where each $x_{i}$ is the weight allocated to the $i$ th asset. Here $\overline{\mathbf{R}}^{\prime} \mathbf{x}$ and $\mathbf{x}^{\prime} \mathbf{\Sigma} \mathbf{x}$ are the expected return and the variance of the portfolio, respectively. We assume that no asset can be represented by a linear combination of other assets, which implies that $\boldsymbol{\Sigma}$ is positive definite. So, the variance is a strictly convex function of portfolio variables. Also, we assume that not all of the mean returns of the assets are equal. A portfolio $\mathbf{x}$ is feasible if it belongs to the following set:

$$
S=\left\{\mathbf{x} \in \mathbb{R}^{n}: \mathbf{A x}=\mathbf{b}, \mathbf{C x} \geq \mathbf{d}\right\}
$$

where $\mathbf{b} \in \mathbb{R}^{m}, \mathbf{d} \in \mathbb{R}^{p}, \mathbf{A}$ is an $m \times n$, and $\mathbf{C}$ is a $p \times n$ matrix over $\mathbb{R}$.

The Markowitz portfolio selection problem, as a Quadratic Programming (QP) problem, which corresponds to expected return $\rho$ has the following form:

\section{Problem 2.1.}

$$
\begin{array}{ll}
\min _{\mathbf{x}} & \frac{1}{2} \mathbf{x}^{\prime} \boldsymbol{\Sigma} \mathbf{x} \\
\text { s.t. } & \overline{\mathbf{R}}^{\prime} \mathbf{x}=\rho, \\
& \mathbf{x} \in S .
\end{array}
$$

The model for $S=\left\{\mathbf{x} \in \mathbb{R}^{n}: \mathbf{1}^{\prime} \mathbf{x}=1, \mathbf{x} \geq 0\right\}$ is called the standard portfolio selection model, where $\mathbf{1}$ is a vector of ones. Merton [12] considered the unbounded portfolio weight model, i.e. $S=\left\{\mathrm{x} \in \mathbb{R}^{n}: \mathbf{1}^{\prime} \mathbf{x}=1\right\}$, and give an analytical solution for the problem.

By the Karush-Kuhn-Tucker $(\mathrm{K}-\mathrm{K}-\mathrm{T})$ conditions, $X^{*}=\left(x_{1}^{*}, \ldots, x_{n}^{*}\right)^{\prime}$ is a (primal) solution or optimal portfolio of Problem 1, if and only if, there exist vectors $\lambda_{\rho} \in \mathbb{R}, \lambda_{\mathbf{b}} \in \mathbb{R}^{m}$ and $\lambda_{\mathbf{d}} \in \mathbb{R}^{p}$ such that:

$$
\begin{gathered}
\boldsymbol{\Sigma} \mathbf{x}^{*}-\lambda_{\rho} \overline{\mathbf{R}}-\mathbf{A}^{\prime} \lambda_{\mathbf{b}}-\mathbf{C}^{\prime} \lambda_{\mathbf{d}}=0 \\
\overline{\mathbf{R}}^{\prime} \mathbf{x}^{*}=\rho, \quad \quad \mathbf{A} \mathbf{x}^{*}=\mathbf{b}, \quad \lambda_{\mathbf{d}}^{\prime}\left(\mathbf{C x}^{*}-\mathbf{d}\right)=0, \\
\mathbf{C x}^{*} \geq \mathbf{d}, \quad \lambda_{\mathbf{d}} \geq 0
\end{gathered}
$$


We denote the primal-dual solution of Problem 1 by $\left(\mathbf{x}^{*}, \lambda_{\rho}, \lambda_{\mathbf{b}}, \lambda_{\mathbf{d}}\right)$ or $\left(\mathbf{x}^{*}, \Lambda\right)$, where $\Lambda:=\left(\lambda_{\rho}, \lambda_{\mathbf{b}}, \lambda_{\mathbf{d}}\right)$. Since variance is strictly convex, $\mathbf{x}^{*}$ is unique. Let us denote the set of all primal-dual solutions of Problem 1 by $\Omega(\rho)$.

Let $\rho_{\text {min }}$ be the expected return of the Global Minimum-Variance (GMV) portfolio which has the minimum variance between all feasible portfolios. We assume that $r_{f}<\rho_{\min }$, where $r_{f}$ is the return of the riskless asset. Also let $\rho_{\max }$ be the highest obtainable expected return of feasible portfolios and $\rho_{T}$ be the expected return of the tangency portfolio. Actually, any efficient portfolio has expected return $\rho \in\left[\rho_{\min }, \rho_{\max }\right]$. As in [17], we define function $\sigma:\left[\rho_{\min }, \rho_{\max }\right] \rightarrow \mathbb{R}$ by $\sigma(\rho):=\left(\mathbf{x}^{* \prime} \boldsymbol{\Sigma} \mathbf{x}^{*}\right)^{1 / 2}$; where $\left(\mathbf{x}^{*}, \Lambda\right) \in \Omega(\rho)$. In fact $\sigma(\rho)$ represents the efficient frontier. Note that $\sigma(\rho)$ is convex but not necessarily smooth. In fact the efficient frontier might have kinks, i.e., the points of nondifferentiability (see $[2,4,18]$ ). Considering this, we refer to $\partial \sigma(\rho)$, as the subdifferential of $\sigma$ at $\rho$, and

$$
L(R)=\left\{\frac{\lambda_{\rho}}{\sigma(\rho)}:\left(\mathbf{x}^{*}, \lambda_{\rho}, \lambda_{\mathbf{b}}, \lambda_{\mathbf{d}}\right) \in \Omega(\rho)\right\},
$$

as stated in [17]. Now the following key theorem holds:

Theorem 2.2. $L(R)=\partial \sigma(\rho)$. Also, $\sigma\left(\rho_{T}\right) /\left(\rho_{T}-r_{f}\right)$, the slope of the optimal $C A L$, belongs to $\partial \sigma\left(\rho_{T}\right)$.

Proof. See Theorem 1 of [17] and the paragraph following the theorem.

For $\left(\mathbf{x}^{*}, \Lambda\right) \in \Omega(\rho)$ we define

$$
\theta(\Lambda):=r_{f} \lambda_{\rho}+\mathbf{b}^{\prime} \lambda_{\mathbf{b}}+\mathbf{d}^{\prime} \lambda_{\mathbf{d}}
$$

The next theorem states some results derived in [17].

Theorem 2.3. For any expected return $\rho \in\left(\rho_{T}, \rho_{\max }\right], \theta(\Lambda)<0$; and for any expected return $\rho \in\left[\rho_{\text {min }}, \rho_{T}\right), \theta(\Lambda)>0$. Also if $\theta(\Lambda)=0$ then, $\rho=\rho_{T}$. Moreover if $\theta(\Lambda)>0(\theta(\Lambda)<0)$ for $\rho=\rho_{\max }\left(\rho=\rho_{\min }\right)$ then $\rho_{\max }=\rho_{T}\left(\rho_{\min }=\rho_{T}\right)$.

Proof. See Corollary 1 of [17] and the paragraph following the corollary.

The assertions of Theorem 2.3 are presented with respect to corresponding Lagrangian multiplier of any optimal portfolio. We use the above theorem to get similar results with respect to optimal asset weights.

\section{The MODIFIED PROBLEM}

In this section, we introduce the modified problem that corresponds to Problem 1 , which contains an additional variable $x_{f}$ so-called free variable. In this case the new portfolio is $\mathbf{x}_{1}:=\left(x_{1}, \ldots, x_{n}, x_{f}\right)^{\prime} \in \mathbb{R}^{n+1}$. In the following we show $\mathbf{x}_{1}$ by $\left(\mathbf{x}, x_{f}\right)$ where $\mathbf{x}=\left(x_{1}, \ldots, x_{n}\right)^{\prime}$. 
Considering Problem 1, the modified portfolio selection problem for the expected return $\rho$ is:

\section{Problem 3.1.}

$$
\begin{array}{ll}
\min _{\mathbf{x}_{1}} & \frac{1}{2} \mathbf{x}_{1}^{\prime} \boldsymbol{\Sigma}_{1} \mathbf{x}_{1} \\
\text { s.t. } & \overline{\mathbf{R}}_{1}^{\prime} \mathbf{x}_{1}=\rho, \\
& \mathbf{x}_{\mathbf{1}} \in S_{1},
\end{array}
$$

where

$$
S_{1}=\left\{\mathbf{x}_{\mathbf{1}} \in \mathbb{R}^{n+1}: \mathbf{A}_{1} \mathbf{x}_{1}=\mathbf{b}, \mathbf{C}_{1} \mathbf{x}_{1} \geq \mathbf{d}, x_{f} \geq 0\right\}, \quad \mathbf{A}_{1}=(\mathbf{A} \mathbf{b})
$$

and

$$
\mathbf{C}_{1}=\left(\begin{array}{ll}
\mathbf{C ~ d}
\end{array}\right), \quad \overline{\mathbf{R}}_{1}=\left(\begin{array}{l}
\overline{\mathbf{R}} \\
r_{f}
\end{array}\right)
$$

and

$$
\boldsymbol{\Sigma}_{1}=\left(\begin{array}{cc}
\boldsymbol{\Sigma} & 0 \\
0 & c
\end{array}\right)
$$

for the arbitrary constant $c>0$. (The value of $c$ can be interpreted as the variance of the return of the asset $x_{f}$ which its return is uncorrelated with the returns of the other assets and has the expected value $r_{f}$ ). The last two constraints in $S_{1}$ can be replaced by $\mathbf{C}_{2} \mathbf{x}_{1} \geq \mathbf{d}_{1}$, where

$$
\mathbf{C}_{2}=\left(\begin{array}{cc}
\mathbf{C} & \mathbf{d} \\
0 & 1
\end{array}\right) \quad \text { and } \quad \mathbf{d}_{1}=\left(\begin{array}{c}
\mathbf{d} \\
0
\end{array}\right) .
$$

$\mathbf{x}_{1} \in S_{1}$ is called a feasible portfolio. Obviously $\mathbf{x}$ satisfies the conditions of Problem 1 , if and only if, $(\mathbf{x}, 0)$ satisfies the conditions of Problem 2.

Let $\mathbf{x}_{1}^{*}=\left(\hat{\mathbf{x}}, x_{f}^{*}\right)$ and $\left(\hat{\mathbf{x}}, x_{f}^{*}, \hat{\Lambda}\right):=\left(\hat{\mathbf{x}}, x_{f}^{*}, \hat{\lambda}_{\rho}, \hat{\lambda}_{\mathbf{b}}, \hat{\lambda}_{\mathbf{d}}, \hat{\lambda}_{f}\right)$ denote the (primal) solution and a primal-dual solution of problem 2 , respectively, then

$$
\begin{gathered}
\mathbf{\Sigma} \hat{\mathbf{x}}-\hat{\lambda}_{\rho} \overline{\mathbf{R}}-\mathbf{A}^{\prime} \hat{\lambda}_{\mathbf{b}}-\mathbf{C}^{\prime} \hat{\lambda}_{\mathbf{d}}=0, \\
c x_{f}^{*}-\bar{r}_{f} \hat{\lambda}_{\rho}-\mathbf{b}^{\prime} \hat{\lambda}_{\mathbf{b}}-\mathbf{d}^{\prime} \hat{\lambda}_{\mathbf{d}}=\hat{\lambda}_{f}, \\
\overline{\mathbf{R}}_{1}^{\prime} \mathbf{x}_{1}^{*}=\rho, \quad \mathbf{A}_{1} \mathbf{x}_{1}^{*}=\mathbf{b}, \quad \hat{\lambda}_{\mathbf{d}}^{\prime}\left(\mathbf{C}_{1} \mathbf{x}_{1}^{*}-\mathbf{d}\right)=0, \quad \hat{\lambda}_{f} x_{f}^{*}=0, \\
\mathbf{C}_{1} \mathbf{x}_{1}^{*} \geq \mathbf{d}, \quad x_{f}^{*} \geq 0, \quad \hat{\lambda}_{\mathbf{d}} \geq 0, \quad \hat{\lambda}_{f} \geq 0 .
\end{gathered}
$$

We denote the set of all primal-dual solution of Problem 2 by $\hat{\Omega}(\rho)$ and define

$$
\hat{\theta}(\hat{\Lambda}):=r_{f} \hat{\lambda}_{\rho}+\mathbf{b}^{\prime} \hat{\lambda}_{\mathbf{b}}+\mathbf{d}_{1}^{\prime} \hat{\lambda}_{I}
$$

where $\left(\hat{\mathbf{x}}, x_{f}^{*}, \hat{\Lambda}\right) \in \hat{\Omega}(\rho)$ and $\hat{\lambda}_{I}=\left(\hat{\lambda}_{\mathbf{d}}^{\prime}, \hat{\lambda}_{f}\right)^{\prime}$. Note that $\hat{\theta}(\hat{\Lambda})=r_{f} \hat{\lambda}_{\rho}+\mathbf{b}^{\prime} \hat{\lambda}_{\mathbf{b}}+\mathbf{d}^{\prime} \hat{\lambda}_{\mathbf{d}}$.

Let $\hat{\rho}_{\max }$ and $\hat{\rho}_{\min }$ be the maximum and the minimum obtainable expected returns, respectively, and $\hat{\rho}_{T}$ be the mean return of the tangency portfolio related to Problem 2. Then the following theorem holds:

Theorem 3.2. If $\rho \in\left[\hat{\rho}_{T}, \hat{\rho}_{\max }\right]$ then, $x_{f}^{*}=0$. Also, $x_{f}^{*}>0$ for any $\rho \in$ $\left[\hat{\rho}_{\min }, \hat{\rho}_{T}\right)$. 


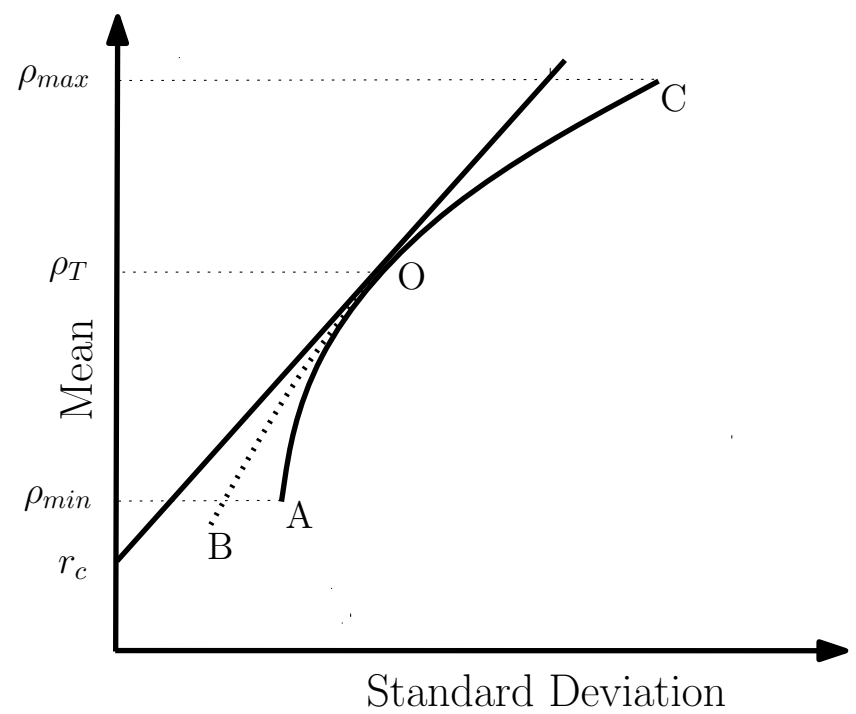

Figure 1. The efficient frontiers of Problem 1 and Problem 2 are the solid curve AOC and the doted-solid curve BOC, respectively.

Proof. Let $\rho=\hat{\rho}_{T}$. Theorem 2.2 implies that there exists a primal-dual solotion $\left(\hat{\mathbf{x}}, x_{f}^{*}, \hat{\lambda}_{\hat{\rho}_{T}}, \hat{\lambda}_{\mathbf{b}}, \hat{\lambda}_{\mathbf{d}}, \hat{\lambda}_{f}\right) \in \hat{\Omega}\left(\hat{\rho}_{T}\right)$ such that

$$
\frac{\hat{\lambda}_{\hat{\rho}_{T}}}{\hat{\sigma}\left(\hat{\rho}_{T}\right)}=\frac{\hat{\sigma}\left(\hat{\rho}_{T}\right)}{\hat{\rho}_{T}-r_{f}},
$$

where $\hat{\sigma}\left(\hat{\rho}_{T}\right)$ is the standard deviation of tangency portfolio. Observe that

$$
\hat{\lambda}_{\hat{\rho}_{T}}\left(\hat{\rho}_{T}-r_{f}\right)=\hat{\mathbf{x}}^{\prime} \boldsymbol{\Sigma} \hat{\mathbf{x}}^{\prime}+c x_{f}^{* 2}=\hat{\rho}_{T} \hat{\lambda}_{\hat{\rho}_{T}}+\mathbf{b}^{\prime} \hat{\lambda}_{\mathbf{b}}+\mathbf{d}^{\prime} \hat{\lambda}_{\mathbf{d}},
$$

where the last equation is obtained by adding the two first equations of (3.1) multiplied by $\hat{\mathbf{x}}^{\prime}$ and $x_{f}^{*}$, respectively. Thus, $\hat{\theta}(\hat{\Lambda})=0$. Now the second equation of (3.1) and the condition $\hat{\lambda}_{f} x_{f}^{*}=0$ shows that $x_{f}^{*}=\hat{\lambda}_{f}=0$.

Now let $\rho \in\left(\hat{\rho}_{T}, \hat{\rho}_{\max }\right]$. By Theorem 2.3 we have $\hat{\theta}(\hat{\Lambda})<0$. Again $x_{f}^{*}=0$ and $\hat{\lambda}_{f}=-\hat{\theta}(\hat{\Lambda})>0$, by the second equation of (3.1) and the condition $\hat{\lambda}_{f} x_{f}^{*}=0$. If $\rho \in\left[\hat{\rho}_{\text {min }}, \hat{\rho}_{T}\right)$ then, Theorem 2.3 implies that $\hat{\theta}(\hat{\Lambda})>0$ and we conclude that $x_{f}^{*}=\hat{\theta}(\hat{\Lambda}) / c>0$ and $\hat{\lambda}_{f}=0$.

Note. Theorem 3.2 implies that Problems 1 and 2 are equivalent for any $\rho \in\left[\hat{\rho}_{T}, \hat{\rho}_{\max }\right]$, in the sense that both problems have the same optimal portfolio, and consequently have the same efficient frontier (see Fig. 1). Indeed, if $\left(\hat{\mathbf{x}}, 0, \hat{\lambda}_{\rho}, \hat{\lambda}_{\mathbf{b}}, \hat{\lambda}_{\mathbf{d}}, \hat{\lambda}_{f}\right) \in \hat{\Omega}(\rho)$ then $\left(\hat{\mathbf{x}}, \hat{\lambda}_{\rho}, \hat{\lambda}_{\mathbf{b}}, \hat{\lambda}_{\mathbf{d}}\right) \in \Omega(\rho)$. Also, the efficient frontier of Problem 2 strictly dominates the efficient frontier of Problem 1 when $\rho<\hat{\rho}_{T}$. 
Corollary 3.3. $\rho_{\max }=\hat{\rho}_{\max }$, where both of the problems 1 and 2 have the same optimal portfolio. Also, $\rho_{\min } \leq \hat{\rho}_{T}$.

Proof. Since $\mathbf{x}$ is feasible if and only if $\mathbf{x}_{1}=(\mathbf{x}, 0)$ is feasible, $\rho_{\max } \leq \hat{\rho}_{\max }$. On the contrary, let $\rho_{\max }<\hat{\rho}_{\max }$. Also let $\left(\hat{\mathbf{x}}, x_{f}^{*}, \hat{\Lambda}\right) \in \hat{\Omega}\left(\hat{\rho}_{\max }\right)$. By Theorem 3.2, we have $x_{f}^{*}=0$. So $\overline{\mathbf{R}}^{\prime} \hat{\mathbf{x}}=\hat{\rho}_{\max }$ which contradicts the maximality of $\rho_{\max }$. Also, if we let $\hat{\rho}_{T}<\rho_{\min }$ and $(\hat{\mathbf{x}}, 0, \hat{\Lambda}) \in \hat{\Omega}\left(\hat{\rho}_{T}\right)$ then, $\overline{\mathbf{R}}^{\prime} \hat{\mathbf{x}}<\rho_{\min }$ which is impossible.

Corollary 3.4. $\hat{\rho}_{T}=\rho_{T}$, where both of the problems 1 and 2 have the same tangency portfolio (see Fig. 1).

Proof. From corollary 3.3 we conclude that $\rho_{\min } \leq \hat{\rho}_{T} \leq \rho_{\max }$. Since problems 1 and 2 have the same efficient frontiers on $\left[\hat{\rho}_{T}, \rho_{\max }\right]$ and the efficient frontier of Problem 2 strictly dominates the efficient frontier of Problem 1 on $\left[\rho_{\min }, \hat{\rho}_{T}\right)$, the optimal CAL corresponding to Problem 2 which is tangent to the efficient frontier of the problem, is also tangent to the efficient frontier of Problem 1.

Note. The results of Theorem 3.2 and its corollaries are independent of $c$.

\section{The TAngency PORTFOlio AS A CORner PORTFOlio}

In this section we discuss some advantages of applying Problem 2 and describe our algorithm. Indeed, we can locate the expected return of the tangency portfolio associated with Problem 1, which is also the tangency portfolio associated with Problem 2 (Cor. 3.4), by looking at the value of free variable in the solution of Problem 2. Similar to the first step, this method can be used to compare the location of $\rho_{T}$ with respect to an arbitrary expected return $\rho$. After finding the optimal portfolio $\left(\hat{\mathbf{x}}, x_{f}^{*}\right)$ (obtained from any QP algorithm) for expected return $\rho$, we will have:

- If $x_{f}^{*}=0$ then, $\rho_{T} \leq \rho$;

- If $x_{f}^{*}>0$ then, $\rho_{T}>\rho$.

Obviously the first case inexplicitly says that, if $\rho=\rho_{\min }$ and $x_{f}^{*}=0$, then $\rho_{T}=\rho_{\text {min }}$. But, we can take more main steps to determine the exact location of $\rho_{T}$ as described in the following.

A M-V portfolio selection problem can be considered as a bi-objective problem as follows:

$$
\min \left\{\mathbf{x}^{\prime} \mathbf{\Sigma} \mathbf{x}\right\} \quad \max \left\{\overline{\mathbf{R}}^{\prime} \mathbf{x}\right\} \quad \text { s.t. } \mathbf{x} \in S .
$$

Here, a feasible portfolio $\mathbf{x}$ is efficient if there exists no feasible portfolio $\mathbf{y}$ such that $\overline{\mathbf{R}}^{\prime} \mathbf{y} \geq \overline{\mathbf{R}}^{\prime} \mathbf{x}$ and $\mathbf{y}^{\prime} \boldsymbol{\Sigma} \mathbf{y} \geq \mathbf{x}^{\prime} \boldsymbol{\Sigma} \mathbf{x}$ with at least one strict inequality. Model (4.1) can be operationalized as

$$
\min _{\mathbf{x}} \mathbf{x}^{\prime} \mathbf{\Sigma} \mathbf{x}-\lambda \overline{\mathbf{R}}^{\prime} \mathbf{x} \quad \text { s.t. } \mathbf{x} \in S
$$

where $\lambda \in[0, \infty)$. PQP algorithms are employed in order to solve (4.2) and compute the efficient frontier as well as all corner portfolios, which will be described 
here. A linear inequality $f(\mathbf{x}) \leq b$ is active at $\mathbf{x}$ if $f(\mathbf{x})=b$, otherwise, i.e. $f(\mathbf{x})<b$, the constraint is inactive. Equations are considered as constraints that are always active. The set of all active constraints at a given point is called the active set. An efficient portfolio is said to be a corner portfolio if in its vicinity on the efficient frontier, other efficient portfolios have different active sets, see [15]. The efficient frontier is made up by a series of connected hyperbolic segment in the M-SD space for which corner portfolios correspond to the endpoints of each segment. The GMV portfolio, however, is included as a corner portfolio (last corner portfolio) irrespective of its active set.

\subsection{Description of the Algorithm}

Note that, since $x_{f}^{*}=0$ for any $\rho \geq \rho_{T}$ and $x_{f}^{*}>0$ for any $\rho<\rho_{T}$, the tangency portfolio is a corner portfolio in the modified problem. Now, we may use any relevant $\mathrm{PQP}$ algorithm, which produce corner portfolios, to determine the tangency portfolio as a corner portfolio. We describe the algorithm for CLA. Our model works as follows: First construct Problem 2 that corresponds to Problem 1 and then apply CLA. Follow the iterates of the algorithm until it admits the first corner portfolio where $x_{f}^{*}$ appears in the optimal portfolios following the corner portfolio. Now this corner portfolio is the tangency portfolio. If $x_{f}^{*}$ is zero throughout the method, then GMV portfolio is the tangency portfolio.

Tütüncü [17] presented an algorithm using CLA to calculate the tangency portfolio. For this, he used the Lagrangian multipliers generated by the CLA and applied the sign of $\theta(\Lambda)$ during the iterations of the method. Precisely, if at the beginning $\left(\rho=\rho_{\max }\right), \theta(\Lambda)>0$, then $\rho_{T}=\rho_{\max }$. Otherwise, as soon as $\theta(\Lambda)$ takes nonnegative values during the iterations of the CLA, then the tangency portfolio is a convex combination of the last and the current corner portfolios and can be obtained by using linear interpolation. Finally, if $\theta(\Lambda)$ is negative throughout the method then, $\rho_{T}=\rho_{\min }$.

Compared to Tütüncü's model, our method does not require any redundant computation such as linear interpolation; and the tangency portfolio can be obtained as a corner portfolio very fast. As mentioned before, for example, the algorithm of Niedermayer and Niedermayer [13] can compute the efficient frontier of the standard problem with 2000 assets in less than a second. Besides, our model works directly with the optimal asset weights, instead of the Lagrangian multipliers, which is more clear to express, and also can employ other suitable PQP algorithms.

Moreover, in a M-SD portfolio selection problem for risky assets and a riskless asset, if borrowing of the riskless asset is not allowed, then the CLA (applied to Prob. 2), generates all (totally) risky efficient portfolios beyond the tangency portfolio (the algorithm can be stopped when it admits the tangency portfolio). The remaining efficient portfolios lie on the optimal CAL. Now, using this method, we can compute the efficient frontier of the problem. 


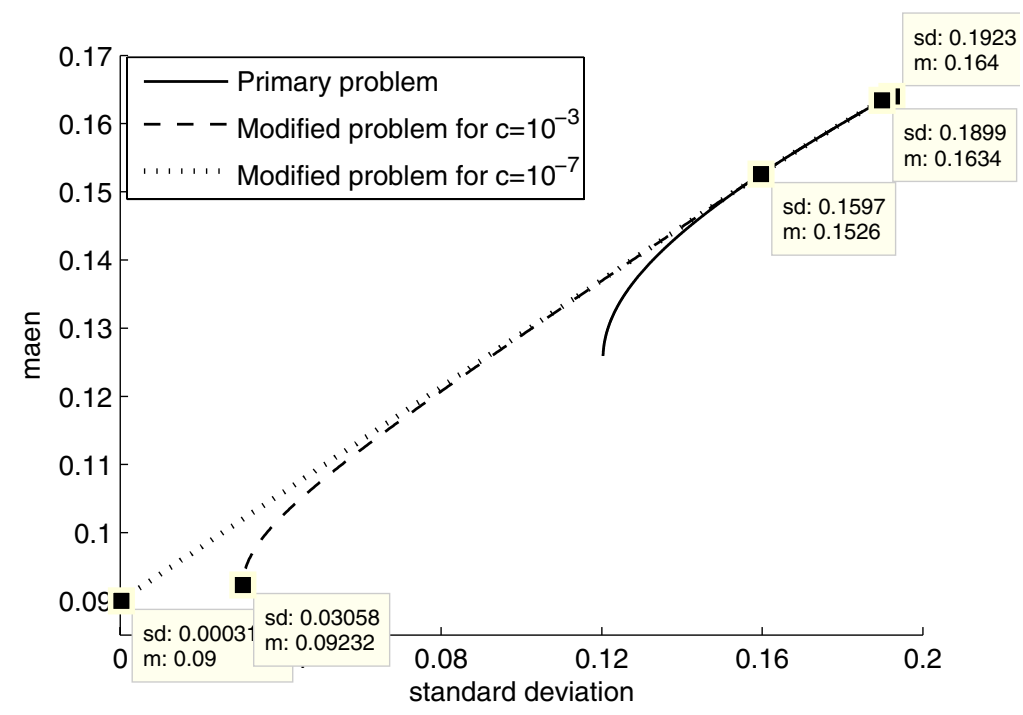

FiguRE 2. Efficient frontiers corresponding to Example 4.1. Black squares denote the location of corner portfolios.

Example 4.1. Consider a portfolio selection problem for three risky assets with expected return vector $\overline{\mathbf{R}}=(0.120 .130 .17)^{\prime}$ and covariance matrix $\boldsymbol{\Sigma}=$ $\left(\begin{array}{ccc}0.015 & 0.012 & 0.011 \\ 0.012 & 0.085 & 0.01 \\ 0.011 & 0.01 & 0.045\end{array}\right)$. We consider 0 and 0.85 to be the lower and the upper bounds for all assets, i.e., $0 \leq x_{i} \leq 0.85$ for $i=1,2,3$. We set $r_{f}=0.09$. Now the modified problem is:

$$
\min \left\{\mathbf{x}_{1}^{\prime} \boldsymbol{\Sigma}_{1} \mathbf{x}_{1}\right\} \quad \max \left\{\overline{\mathbf{R}}_{1}^{\prime} \mathbf{x}_{1}\right\} \quad \text { s.t. } \mathbf{x}_{1} \in S_{1}
$$

where $S_{1}=\left\{\mathbf{x}_{1} \in \mathbb{R}^{4}: \mathbf{1}^{\prime} \mathbf{x}_{1}=1, \mathbf{C}_{1} \mathbf{x}_{1} \leq \mathbf{d}, \mathbf{x}_{1} \geq \mathbf{0}\right\}, \mathbf{C}_{1}=\left(\begin{array}{llll}1 & 0 & 0 & 0.85 \\ 0 & 1 & 0 & 0.85 \\ 0 & 0 & 1 & 0.85\end{array}\right)$ and $\mathbf{d}=\left(\begin{array}{lll}0.85 & 0.85 & 0.85\end{array}\right)^{\prime}$. We use Optimizer to compute the corner portfolios. The efficient frontiers are computed for $c=10^{-3}$ and $10^{-7}$ (see Fig. 2) and corner portfolios are presented in Table 1 . As it is shown in Table 1, $\mathbf{x}_{1}^{3}$ is the last corner portfolio for which free variable dose not appear in corner portfolios. So $\mathbf{x}_{1}^{3}$ is the tangency portfolio. Also when portfolio contains a riskless asset with $r_{f}=0.09$ and borrowing is not allowed, the efficient frontier contains $\mathbf{x}_{1}^{1}, \mathbf{x}_{1}^{2}, \mathbf{x}_{1}^{3}$ and $\mathbf{x}_{1}^{0}$ as the corner portfolios, where $\mathbf{x}_{1}^{0}$ corresponds to the totally riskless investment, i.e., $(0,0.09)$, on the mean axis. Moreover, for values of $c$ closed to zero, GMV portfolio acts like a totally riskless portfolio and that segment of the efficient frontier between the tangency portfolio and the GMV portfolio, tends to a straight line. 
TABLE 1. Efficient extreme points corresponding to Example 4.1.

\begin{tabular}{l|cccc|cccc}
\hline & \multicolumn{5}{|c|}{$c=10^{-3}$} & \multicolumn{3}{c}{$c=10^{-7}$} \\
Corner portfolio & $x_{1}$ & $x_{2}$ & $x_{3}$ & $x_{4}$ & $x_{1}$ & $x_{2}$ & $x_{3}$ & $x_{4}$ \\
\hline $\mathbf{x}_{1}^{1}$ & 0.000 & 0.150 & 0.850 & 0.000 & 0.000 & 0.150 & 0.850 & 0.000 \\
$\mathbf{x}_{1}^{2}$ & 0.058 & 0.092 & 0.850 & 0.000 & 0.058 & 0.092 & 0.850 & 0.000 \\
$\mathbf{x}_{1}^{3}$ & 0.286 & 0.076 & 0.637 & 0.000 & 0.286 & 0.076 & 0.637 & 0.000 \\
$\mathbf{x}_{1}^{4}$ & 0.056 & 0.002 & 0.007 & 0.935 & 0.000 & 0.000 & 0.000 & 1.000 \\
\hline
\end{tabular}

So, the obtained efficient frontier can be consider as a good approximation for the original efficient frontier for which the portfolio contains the three risky assets and the riskless asset.

\section{REFERENCES}

[1] M.J. Best, An algorithm for the solution of the parametric quadratic programming problem, in Applied mathematics and parallel computing: Festchrift for KLaus Ritter, H. Fischer, B. Riedmüller, S. Schäfler, eds. Physica-verlag (1996) 57-76.

[2] M.J. Best and R.R. Grauer, The efficient set mathematics when the mean variance problem is subject to general linear constraints. J. Econ. Bus. 42 (1990) 105-120.

[3] Z. Bodie, A. Kane and A.J. Marcus, Investments, 8rd edn. McGraw-Hill Irwin, New York (2009).

[4] P.H. Dybvig, Short sales restrictions and kinks on the mean variance frontier. J. Finance 39 (1984) 239-244.

[5] M. Hirschberger, Y. Qi and R.E. Steuer, Large-scale MV efficient frontier computation via a procedure of parametric quadratic programming. Eur. J. Oper. Res. 204 (2010) 581-588.

[6] B.I. Jacobs, K.N. Levy and H.M. Markowitz, Portfolio optimization with factors, scenarios, and realistic short positions. Oper. Res. 53 (2005) 586-599.

[7] H.M. Markowitz, Portfolio selection. J. Finance 7 (1952) 77-91.

[8] H.M. Markowitz, The optimization of a quadratic function subject to linear constraints. Naval Res. Logist. Quarterly 3 (1956) 111-33.

[9] H.M. Markowitz, Portfolio selection: Efficient diversification of investments. John Wiley, New York (1959).

[10] H.M. Markowitz, Mean-variance analysis in portfolio choice and capital markets. Basil Blackwell, Oxford, UK (1987).

[11] H.M. Markowitz and P. Todd, Mean-variance analysis in portfolio choice and capital markets. Frank J. Fabozzi Associates, New Hope, Pennsylvania (2000).

[12] R.C. Merton, An analytical derivation of the efficient portfolio frontier. J. Financial Quant. Anal. 7 (1972) 1851-1872.

[13] A. Niedermayer and D. Niedermayer, Applying Markowitz's critical line algorithm, in Handbook of portfolio construction, J.B. Guerard (Ed.), Springer-Verlag, Berlin (2010) 383-400.

[14] W.F. Sharpe, The Sharpe ratio. J. Portfolio Manage. Fall (1994) 49-58.

[15] M. Stein, J. Branke and H. Schmeck, Efficient implementation of an active set algorithm for large-scale portfolio selection. Comput. Oper. Res. 35 (2008) 3945-3961.

[16] J. Tobin, Liquidity preference as a behavior towards risk. Rev. Econ. Stud. 25 (1958) 65-86.

[17] R.H. Tütüncü, A note on calculating the optimal risky portfolio. Finance Stoch. 5 (2001) 413-417.

[18] J. Vörös, J. Kriens and L.W.G. Strijbosch, A note on the kinks at the mean variance frontier. Eur. J. Oper. Res. 112 (1999) 236-239. 